
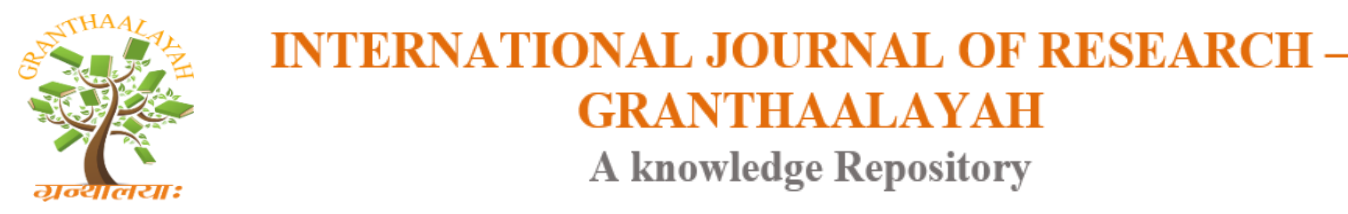

Science

\title{
REAL POWER LOSS REDUCTION ENHANCED ARTIFICIAL BEE COLONY ALGORITHM
}

\author{
Dr.K. Lenin *1 \\ ${ }^{* 1}$ Professor, Department of EEE, Prasad V.Potluri Siddhartha Institute of Technology, Kanuru, \\ Vijayawada, Andhra Pradesh -520007, India
}

\begin{abstract}
In this paper, Enhanced Artificial Bee Colony (EABC) algorithm is proposed for solving optimal reactive power problem. The projected method assimilates crossover operation from Genetic Algorithm (GA) with artificial bee colony (ABC) algorithm. The EABC strengthens the exploitation phase of $\mathrm{ABC}$ as crossover enhances exploration of search space. Projected EABC algorithm has been tested on has been tested on standard IEEE 118 \& practical 191 bus test systems and simulation results show clearly about the premium performance of the proposed algorithm in reducing the real power loss.
\end{abstract}

Keywords: Optimal Reactive Power; Transmission Loss; Artificial Bee Colony Algorithm; Genetic Algorithms; Crossover Operator; Particle Swarm Optimization.

Cite This Article: Dr.K. Lenin. (2018). "REAL POWER LOSS REDUCTION ENHANCED ARTIFICIAL BEE COLONY ALGORITHM." International Journal of Research Granthaalayah, 6(3), 203-213. https://doi.org/10.29121/granthaalayah.v6.i3.2018.1515.

\section{Introduction}

Optimal reactive power problem is to minimize the real power loss and bus voltage deviation. Various numerical methods like the gradient method [1-2], Newton method [3] and linear programming [4-7] have been adopted to solve the optimal reactive power dispatch problem. Both the gradient and Newton methods have the complexity in managing inequality constraints. If linear programming is applied then the input- output function has to be uttered as a set of linear functions which mostly lead to loss of accuracy. The problem of voltage stability and collapse play a major role in power system planning and operation [8]. Evolutionary algorithms such as genetic algorithm have been already proposed to solve the reactive power flow problem [9-11]. Evolutionary algorithm is a heuristic approach used for minimization problems by utilizing nonlinear and non-differentiable continuous space functions. In [12], Hybrid differential evolution algorithm is proposed to improve the voltage stability index. In [13] Biogeography Based algorithm is projected to solve the reactive power dispatch problem. In [14], a fuzzy based method is used to solve the optimal reactive power scheduling method. In [15], an improved evolutionary programming is used to solve the optimal reactive power dispatch problem. In [16], 
the optimal reactive power flow problem is solved by integrating a genetic algorithm with a nonlinear interior point method. In [17], a pattern algorithm is used to solve ac-dc optimal reactive power flow model with the generator capability limits. In [18], F. Capitanescu proposes a two-step approach to evaluate Reactive power reserves with respect to operating constraints and voltage stability. In [19], a programming based approach is used to solve the optimal reactive power dispatch problem. In [20], A. Kargarian et al present a probabilistic algorithm for optimal reactive power provision in hybrid electricity markets with uncertain loads. In this paper, Enhanced Artificial bee colony (EABC) algorithm is proposed for solving optimal reactive power problem. ABC (Artificial Bee Colony) algorithm was proposed by Dervis Karaboga in 2005, which is based on the intelligent behaviour of honeybee swarms finding nectar and sharing the information of food sources with each other [21]. ABC algorithm has the advantages of strong robustness, fast convergence and high flexibility, fewer control parameters. ABC algorithm can be used for solving multidimensional and multimodal optimization problems [2224]. However, it has been shown that the ABC may infrequently stop moving toward the global optimum even though the population has not encounter to a local optimum [25]. It can be realistic that the solution search equation of $\mathrm{ABC}$ algorithm is good at exploration but poor at exploitation [26]. Therefore, to uphold the appropriate balance between exploration and exploitation behaviour of $\mathrm{ABC}$, it is highly expected to build up a local search approach in the basic $A B C$ to strengthen the search region. So in this work Genetic algorithm integrated with artificial bee algorithm to improve the efficiency of the process, such that it will lead to reach a global solution for our optimal reactive power dispatch problem. Projected EABC algorithm has been tested on has been tested on standard IEEE 118 \& practical 191 bus test systems and simulation results show clearly about the premium performance of the proposed algorithm in reducing the real power loss.

\section{Problem Formulation}

The objective of the optimal reactive power problem is to minimize one or more objective functions while satisfying a number of constraints such as load flow, generator bus voltages, load bus voltages, switchable reactive power compensations, reactive power generation, transformer tap setting and transmission line flow.

\subsection{Minimization of Real Power Loss}

It is aimed in this objective that minimizing of the real power loss (Ploss) in transmission lines of a power system. This is mathematically stated as follows.

$P_{\text {loss }}=\sum_{k=(i, j)}^{n} g_{k=1}\left(V_{i}^{2}+V_{j}^{2}-2 V_{i} V_{j} \cos \theta_{i j}\right)$

Where $\mathrm{n}$ is the number of transmission lines, $\mathrm{g}_{\mathrm{k}}$ is the conductance of branch $\mathrm{k}, \mathrm{V}_{\mathrm{i}}$ and $\mathrm{V}_{\mathrm{j}}$ are voltage magnitude at bus $i$ and bus $j$, and $\theta_{\mathrm{ij}}$ is the voltage angle difference between bus $\mathrm{i}$ and bus j.

\subsection{Minimization of Voltage Deviation}

It is aimed in this objective that minimizing of the 
Deviations in voltage magnitudes (VD) at load buses. This is mathematically stated as follows.

Minimize VD $=\sum_{k=1}^{n l}\left|V_{k}-1.0\right|$

Where $\mathrm{n}_{\mathrm{l}}$ is the number of load busses and $\mathrm{V}_{\mathrm{k}}$ is the voltage magnitude at bus $\mathrm{k}$.

\subsection{System Constraints}

In the minimization process of objective functions, some problem constraints which one is equality and others are inequality had to be met. Objective functions are subjected to these constraints shown below.

Load flow equality constraints:

$P_{G i}-P_{D i}-V_{i \sum_{j=1}^{n b} V_{j}}\left[\begin{array}{cc}G_{i j} & \cos \theta_{i j} \\ +B_{i j} & \sin \theta_{i j}\end{array}\right]=0, i=1,2 \ldots, n b$

$Q_{G i}-Q_{D i} V_{i \sum_{j=1}^{n b} V_{j}}\left[\begin{array}{cc}G_{i j} & \cos \theta_{i j} \\ +B_{i j} & \sin \theta_{i j}\end{array}\right]=0, i=1,2 \ldots, n b$

where, $\mathrm{nb}$ is the number of buses, $\mathrm{P}_{\mathrm{G}}$ and $\mathrm{Q}_{\mathrm{G}}$ are the real and reactive power of the generator, $\mathrm{P}_{\mathrm{D}}$ and $Q_{D}$ are the real and reactive load of the generator, and $G_{i j}$ and $B_{i j}$ are the mutual conductance and susceptance between bus i and bus $j$.

Generator bus voltage $\left(V_{G i}\right)$ inequality constraint:

$V_{G i}^{\min } \leq V_{G i} \leq V_{G i}^{\max }, i \in n g$

Load bus voltage $\left(V_{L i}\right)$ inequality constraint:

$V_{L i}^{\min } \leq V_{L i} \leq V_{L i}^{\max }, i \in n l$

Switchable reactive power compensations $\left(Q_{C i}\right)$ inequality constraint:

$Q_{C i}^{\min } \leq Q_{C i} \leq Q_{C i}^{\max }, i \in n c$

Reactive power generation $\left(Q_{G i}\right)$ inequality constraint:

$Q_{G i}^{\min } \leq Q_{G i} \leq Q_{G i}^{\max }, i \in n g$

Transformers tap setting $\left(T_{i}\right)$ inequality constraint:

$T_{i}^{\min } \leq T_{i} \leq T_{i}^{\max }, i \in n t$

Transmission line flow $\left(\mathrm{S}_{\mathrm{Li}}\right)$ inequality constraint:

$S_{L i}^{\min } \leq S_{L i}^{\max }, i \in n l$

Where, nc, ng and nt are numbers of the switchable reactive power sources, generators and transformers. 


\section{Artificial Bee Colony (ABC) Algorithm}

The artificial bee colony contains three groups: employed bee, onlooker bee and scout. The bee going to the food source which is visited by itself previously is employed bee. The bee waiting on the dance area for making decision to choose a food source is onlooker bee. The bee carrying out random search is scout bee. The onlooker bee with scout also called unemployed bee. In the ABC algorithm, the collective intelligence searching model of artificial bee colony consists of three essential components: employed, unemployed foraging bees, and food sources. The employed and unemployed bees search for the rich food sources, which close to the bee's hive. The employed bees store the food source information and share the information with onlooker bees. The number of employed bees is equal to the number of food sources and also equal to the amount of onlooker bees. Employed bees whose solutions cannot be improved through a predetermined number of trials, specified by the user of the ABC algorithm and called "limit", become scouts and their solutions are abandoned. The model also defines two leading modes of behaviour which are necessary for self-organizing and collective intelligence: recruitment of foragers to rich food sources resulting in positive feedback and abandonment of poorsources by scout causing negative feedback.

\subsection{The Procedure of ABC}

The classical ABC includes four main phases.

Initialization Phase: The food sources, whose population size is SN, are randomly generated by scout bees. The number of Artificial Bee is NP. Each food source $x_{m}$ is a vector to the optimization problem, $\mathrm{x}_{\mathrm{m}}$ has $\mathrm{D}$ variables and $\mathrm{D}$ is the dimension of searching space of the objective function to be optimized. The initiation food sources are randomly produced via the expression (11).

$\mathrm{x}_{\mathrm{m}}=\mathrm{l}_{\mathrm{i}}+\operatorname{rand}(0.1) *\left(\mathrm{u}_{\mathrm{i}}-\mathrm{l}_{\mathrm{i}}\right)$

where $u_{i}$ and $l_{i}$ are the upper and lower bound of the solution space of objective function, rand $(0,1)$ is a random number within the range $[0,1]$.

Employed Bee Phase: A employed bee flies to a food source and finds a new food source within the neighborhood of the food source. The higher quantity food source will be selected. The food source information stored by employed bee will be shared with onlooker bees. A neighbor food source $\mathrm{v}_{\mathrm{mi}}$ is determined and calculated by the following equation (12).

$\mathrm{v}_{\mathrm{mi}}=\mathrm{x}_{\mathrm{mi}}+\Phi_{\mathrm{mi}}\left(\mathrm{x}_{\mathrm{mi}}-\mathrm{x}_{\mathrm{ki}}\right)$

where $x_{k}$ is a randomly selected food source, $i$ is a randomly chosen parameter index, $\Phi_{m i}$ is a random number within the range $[-1,1]$. The range of this parameter can make an appropriate adjustment on specific issues. The fitness of food source is essential in order to find the global optimal. The fitness is calculated by thefollowing formula (13). After that a greedy selection is applied between $\mathrm{x}_{\mathrm{m}}$ and $\mathrm{v}_{\mathrm{m}}$. 
$\mathrm{fit}_{\mathrm{m}}\left(\mathrm{x}_{\mathrm{m}}\right)=\left\{\begin{array}{c}\frac{1}{1+\mathrm{f}_{\mathrm{m}}\left(\mathrm{x}_{\mathrm{m}}\right)}, \mathrm{f}_{\mathrm{m}}\left(\mathrm{x}_{\mathrm{m}}\right)>0 \\ 1+\left|\mathrm{f}_{\mathrm{m}}\left(\mathrm{x}_{\mathrm{m}}\right)\right|, \mathrm{f}_{\mathrm{m}}\left(\mathrm{x}_{\mathrm{m}}\right)<0\end{array}\right\}$

where $f_{m}\left(x_{m}\right)$ is the objective function value of $x_{m}$.

Onlooker Bee Phase: Onlooker bees observe the waggle dance in the dance area and calculate the profitability of food sources, then randomly select a higher food source. After that onlooker bees carry out randomly search in the neighborhood of food source. The quantity of a food source is evaluated by its profitability and the profitability of all food sources. Pm is determined by the formula

$\mathrm{P}_{\mathrm{m}}=\frac{\mathrm{fit}_{\mathrm{m}}\left(\mathrm{x}_{\mathrm{m}}\right)}{\sum_{\mathrm{m}=1}^{\mathrm{SN}} \mathrm{fit}_{\mathrm{m}}\left(\mathrm{x}_{\mathrm{m}}\right)}$

where fit $_{\mathrm{m}}\left(\mathrm{x}_{\mathrm{m}}\right)$ is the fitness of $\mathrm{x}_{\mathrm{m}}$.

Onlooker bees search the neighbourhood of food source according to the expression (15)

$v_{m i}=x_{m i}+\Phi_{m i}\left(x_{m i}-x_{k i}\right)$

Scout Phase: If the profitability of food source cannot be improved and the times of unchanged greater than the predetermined number of trials, which called "limit" and specified by the user of the ABC algorithm, the solutions will be abandoned by scout bees. Then, the scouts start to randomly search the new solutions. If solution $\mathrm{x}_{\mathrm{i}}$ has been abandoned, the new solution $x_{m}$ will be discovered by the scout. The $x_{m}$ is defined by expression (16)

$x_{m}=l_{i}+\operatorname{rand}(0.1) *\left(u_{i}-l_{i}\right)$

Where $x_{m}$ is the new generated food source, rand $(0,1)$ is a random number within the range $[0$, 1], $u_{i}$ and $l_{i}$ are the upper and lower bound of the solution space of objective function.

\subsection{The Main Concepts of ABC Algorithm}

Food sources: According to different problems, the initial food sources are randomly generated in the search space.

- Local optimization strategy: In the employed bee phase, employed bees look for the local optimization value in the neighborhood of food source. Generally, different local search strategies will are used for different problems.

- Random selection strategy in accordance with probability: In the onlooker bee phase, the random selection strategy will be used to looking for local optimization value in the neighborhood of food source and the higher probability solution will be chosen by onlooker bees.

- Feedback strategy: In scout bee phase, food sources which are initially poor or have been made poor by exploitation will be abandoned, this means that if a solution cannot be 
improved and the unchanged times greater than the predetermined "limit" parameter, the new solution will be discovered by the scout using the negative feedback strategy.

- Global optimization strategy: After local optimization and random selection carried out, the global optimization strategy will be used to obtain global optimal value.

\section{Genetic Algorithm Crossover Operator}

The crossover operator is a scheme for producing genetic information from parents; it combines the characters of two parents to form two off-springs, with the possibility that good chromosomes may evaluate better ones. The crossover operator is not regularly imposed to all pairs of parent solution the intermediate generation. An incidental choice is made, where the possibility of crossover being applied depends on probability determined by a crossover rate, known as crossover probability. The crossover operator is most significant part in GAs. It combines portion of good solution to construct new favorable solution. Information involved in one solution mixed with information involved in another solution and the rising solution will either have good quality fitness or stay alive to commutate this information again. If generated two off-springs are the same then crossover operator show strong heritability [27, 28]. Crossover operators play key role in genetic algorithm which combines the characteristic of existing solutions and generate new solutions. The optimization problems depend upon the data they used so they are classified in to two categories. One is based on real data set and another one is based on binary or discrete data set. Crossover operator also considered as binary crossover operators and real coded crossover operators. Two particles distribute their positional information in the search space and a new particle is formed. The particle, is known as laplacian particle, replaces the nastiest performing particle in the swarm. Using this fresh operator, this paper introduces two algorithms namely Laplace Crossover PSO with inertia weight (LXPSO-W) and Laplace Crossover PSO with constriction factor (LXPSO-C) [29]. A. H. Wright suggests a genetic algorithm that uses real parameter vectors as chromosomes, real parameters as genes, and real numbers as alleles [30].

\subsection{Linear Crossover}

Linear crossover [29, 30] is one of the most primitive operator in real coded crossover it develops three solutions from two parents and the best two off-springs substitute parents. Let $\left(x_{1}^{(1, t)}, x_{2}^{(1, t)}, \ldots, x_{n}^{(1, t)}\right)$ and $\left(x_{1}^{(2, t)}, x_{2}^{(2, t)}, \ldots, x_{n}^{(2, t)}\right)$ are two parent solutions of dimension $n$ at generation $t$. Linear crossover develops three offspring from these parents as shown in Eq.(17, 18 and 19) and best two offspring being chosen as off-springs.

$$
\begin{aligned}
& 0.5\left(X_{i}^{(1, t)}+X_{i}^{(2, t)}\right) \\
& \left(1.5 X_{i}^{(1, t)}-0.5 X_{i}^{(2, t)}\right) \\
& \left(-0.5 X_{i}^{(1, t)}+1.5 X_{i}^{(2, t)}\right)
\end{aligned}
$$

Where $\mathrm{i}=1,2, \ldots, \mathrm{n}$ 


\section{Incorporation of Genetic Algorithm (GA) With Artificial Bee Colony (ABC) Algorithm}

Artificial bee colony (ABC) algorithm with crossover works in five different phases: primary phase is initialization of parameters; second phase is employed bee phase to calculate fresh food sources. Third phase is recently introduces crossover phase. This phase maintains equilibrium between diversification and intensification. Crossover phase branch out the population. Fourth phase is onlooker bee phase to perk up solution based on their fitness. Last phase is scout bee phase, this phase search fresh solutions in place of discarded solutions. The general process of algorithms is followed in steps .The first step consists of the assessment of the population using the Artificial Bee Colony. Primary populations generated by ABC are used by employed bees. Following this crossover operators are applied. If crossover criteria or probability satisfied than two arbitrary parents are taken to execute crossover operation on them. After crossover operation fresh off-springs are developed. Worst parent reinstated by best developed offspring if its fitness is better than the worst parent. Crossover operator is applied to two randomly selected parents from current population. Two offspring developed from crossover and worst parent is reinstated by best offspring, other parent remains same. The whole procedure repeats itself until the maximum numbers of cycles are concluded. In our approach crossover applied in each iteration after employed bee phase.

\subsection{Enhanced Artificial Bee Colony (EABC) Algorithm for Solving Reactive Power Problem}

\subsubsection{Initialization Phase}

For $\mathrm{i}=0$ to the maximum no. of food source size do

For $\mathrm{j}=0$ to the problem dimension

Initialize all food sources arbitrarily

End for $\mathrm{j}$

Calculate fitness for all food sources

End for $\mathrm{i}$

Repeat

\subsubsection{Employed Bee Phase}

For $\mathrm{i}=0$ to maximum no. of employed bees

For $\mathrm{j}=0$ to maximum problem dimension

Create new candidate solution

End for $\mathrm{j}$

Calculate fitness values for all new generated candidate solution

If candidate solution is better than the old solution then reinstate old solution with candidate solution

End for $\mathrm{i}$ 


\subsubsection{Crossover Phase}

If crossover criteria satisfies

For $\mathrm{i}=0$ to the maximum no. of food source

Pick two random individuals from the current population for crossover operation.

Apply crossover operation.

New off-springs generated from parents as a result of crossover. Reinstate the worst parent with the best new offspring if it is better.

End for $\mathrm{i}$

\subsubsection{Onlooker Bee Phase}

For $\mathrm{i}=0$ to the maximum no. of onlooker bees

For $\mathrm{j}=0$ to maximum dimension

Produce new candidate solution

End for $\mathrm{j}$

Compute the fitness for new generated candidate solution

If candidate solution has the better fitness values then reinstate old solution

End for $\mathrm{i}$

\subsubsection{Scout Bee Phase}

If any food source worn out

Initialize arbitrarily exhausted food source until maximum cycle number.

\section{Simulation Results}

At first Enhanced Artificial Bee Colony (EABC) algorithm has been tested in standard IEEE 118-bus test system [31].The system has 54 generator buses, 64 load buses, 186 branches and 9 of them are with the tap setting transformers. The limits of voltage on generator buses are 0.95 1.1 per-unit., and on load buses are 0.95 -1.05 per-unit. The limit of transformer rate is $0.9-1.1$, with the changes step of 0.025 . The limitations of reactive power source are listed in Table 1 , with the change in step of 0.01 .

Table 1: Limitation of reactive power sources

\begin{tabular}{|l|l|l|l|l|l|l|l|}
\hline BUS & 5 & 34 & 37 & 44 & 45 & 46 & 48 \\
\hline QCMAX & 0 & 14 & 0 & 10 & 10 & 10 & 15 \\
\hline QCMIN & -40 & 0 & -25 & 0 & 0 & 0 & 0 \\
\hline BUS & 74 & 79 & 82 & 83 & 105 & 107 & 110 \\
\hline QCMAX & 12 & 20 & 20 & 10 & 20 & 6 & 6 \\
\hline QCMIN & 0 & 0 & 0 & 0 & 0 & 0 & 0 \\
\hline
\end{tabular}

The statistical comparison results have been listed in Table 2 and the results clearly show the better performance of proposed EABC approach. 
Table 2: Comparison results

\begin{tabular}{|l|l|l|l|l|}
\hline Active power loss (p.u) & BBO [32] & $\begin{array}{l}\text { ILSBBO/ strategy1 } \\
{[\text { 32] }}\end{array}$ & $\begin{array}{l}\text { ILSBBO/ } \\
\text { strategy1 [32] }\end{array}$ & $\begin{array}{l}\text { Proposed } \\
\text { EABC }\end{array}$ \\
\hline Min & 128.77 & 126.98 & 124.78 & 112.42 \\
\hline Max & 132.64 & 137.34 & 132.39 & 118.24 \\
\hline Average & 130.21 & 130.37 & 129.22 & 116.86 \\
\hline
\end{tabular}

Then Enhanced Artificial Bee Colony (EABC) algorithm the has been tested in practical 191 test system and the following results have been obtained. In Practical 191 test bus system - Number of Generators $=20$, Number of lines $=200$, Number of buses $=191$ Number of transmission lines $=55$. Table 3 shows the optimal control values of practical 191 test system obtained by EABC method. And table 4 shows the results about the value of the real power loss by obtained by Enhanced Artificial Bee Colony (EABC) algorithm.

Table 3: Optimal Control values of Practical 191 utility (Indian) system by EABC method

\begin{tabular}{|c|c|c|c|}
\hline VG1 & 1.100 & VG 11 & 0.900 \\
\hline VG 2 & 0.760 & VG 12 & 1.000 \\
\hline VG 3 & 1.010 & VG 13 & 1.000 \\
\hline VG 4 & 1.010 & VG 14 & 0.900 \\
\hline VG 5 & 1.100 & VG 15 & 1.000 \\
\hline VG 6 & 1.100 & VG 16 & 1.000 \\
\hline VG 7 & 1.100 & VG 17 & 0.900 \\
\hline VG 8 & 1.010 & VG 18 & 1.000 \\
\hline VG 9 & 1.100 & VG 19 & 1.100 \\
\hline VG 10 & 1.010 & VG 20 & 1.100 \\
\hline
\end{tabular}

\begin{tabular}{|c|c|c|c|c|c|}
\hline $\mathrm{T} 1$ & 1.000 & $\mathrm{~T} 21$ & 0.900 & T41 & 0.900 \\
\hline $\mathrm{T} 2$ & 1.000 & T22 & 0.900 & T42 & 0.900 \\
\hline $\mathrm{T} 3$ & 1.000 & $\mathrm{~T} 23$ & 0.900 & T43 & 0.910 \\
\hline $\mathrm{T} 4$ & 1.100 & T24 & 0.900 & T44 & 0.910 \\
\hline $\mathrm{T} 5$ & 1.000 & $\mathrm{~T} 25$ & 0.900 & $\mathrm{~T} 45$ & 0.910 \\
\hline T6 & 1.000 & T26 & 1.000 & T46 & 0.900 \\
\hline $\mathrm{T} 7$ & 1.000 & T27 & 0.900 & T47 & 0.910 \\
\hline $\mathrm{T} 8$ & 1.010 & T28 & 0.900 & $\mathrm{~T} 48$ & 1.000 \\
\hline T9 & 1.000 & T29 & 1.010 & T49 & 0.900 \\
\hline $\mathrm{T} 10$ & 1.000 & T30 & 0.900 & $\mathrm{~T} 50$ & 0.900 \\
\hline $\mathrm{T} 11$ & 0.900 & T31 & 0.900 & T51 & 0.900 \\
\hline $\mathrm{T} 12$ & 1.000 & T32 & 0.900 & T52 & 0.900 \\
\hline $\mathrm{T} 13$ & 1.010 & T33 & 1.010 & T53 & 1.000 \\
\hline T14 & 1.010 & T34 & 0.900 & T54 & 0.900 \\
\hline $\mathrm{T} 15$ & 1.010 & T35 & 0.900 & T55 & 0.900 \\
\hline T19 & 1.020 & T39 & 0.900 & & \\
\hline $\mathrm{T} 20$ & 1.010 & $\mathrm{~T} 40$ & 0.900 & & \\
\hline
\end{tabular}

Table 4: Optimum real power loss values obtained for practical 191 utility (Indian) system by EABC method

\begin{tabular}{|l|l|}
\hline Real power Loss (MW) & EABC \\
\hline Min & 135.076 \\
\hline Max & 139.282 \\
\hline Average & 137.246 \\
\hline
\end{tabular}




\section{Conclusion}

Enhanced Artificial Bee Colony (EABC) algorithm has been successfully applied for optimal reactive power problem. The projected method assimilates crossover operation from Genetic Algorithm (GA) with artificial bee colony (ABC) algorithm. The EABC strengthens the exploitation phase of $\mathrm{ABC}$ as crossover enhances exploration of search space. Projected EABC algorithm has been tested on has been tested on standard IEEE 118 \& practical 191 bus test systems and simulation results show clearly about the premium performance of the proposed algorithm in reducing the real power loss.

\section{References}

[1] O.Alsac, and B. Scott, "Optimal load flow with steady state security", IEEE Transaction. PAS 1973, pp. 745-751.

[2] Lee K Y, Paru Y M, Oritz J L -A united approach to optimal real and reactive power dispatch , IEEE Transactions on power Apparatus and systems 1985: PAS-104 : 1147-1153

[3] A.Monticelli, M .V.F Pereira, and S. Granville, "Security constrained optimal power flow with post contingency corrective rescheduling”, IEEE Transactions on Power Systems: PWRS-2, No. 1, pp.175-182.,1987.

[4] DeebN, Shahidehpur S.M, Linear reactive power optimization in a large power network using the decomposition approach. IEEE Transactions on power system 1990: 5(2) : 428-435

[5] E. Hobson, "Network consrained reactive power control using linear programming", IEEE Transactions on power systems PAS -99 (4), pp 868=877, 1980

[6] K.Y Lee, Y.M Park, and J.L Oritz, "Fuel -cost optimization for both real and reactive power dispatches", IEE Proc; 131C, (3), pp.85-93.

[7] M.K. Mangoli, and K.Y. Lee, "Optimal real and reactive power control using linear programming", Electr.PowerSyst.Res, Vol.26, pp.1-10, 1993.

[8] C.A. Canizares, A.C.Z.de Souza and V.H. Quintana, "Comparison of performance indices for detection of proximity to voltage collapse", vol. 11. no.3, pp.1441-1450, Aug 1996.

[9] S.R.Paranjothi, and K.Anburaja, "Optimal power flow using refined genetic algorithm", Electr.PowerCompon.Syst, Vol. 30, 1055-1063, 2002.

[10] D. Devaraj, and B. Yeganarayana, "Genetic algorithm based optimal power flow for security enhancement", IEE proc-Generation. Transmission and. Distribution; 152, 6 November 2005.

[11] A. Berizzi, C. Bovo, M. Merlo, and M. Delfanti, "A ga approach to compare or pf objective functions including secondary voltage regulation," Electric Power Systems Research, vol. 84, no. 1, pp. $187-194,2012$.

[12] C.-F. Yang, G. G. Lai, C.-H. Lee, C.-T. Su, and G. W. Chang, "Optimal setting of reactive compensation devices with an improved voltage stability index for voltage stability enhancement," International Journal of Electrical Power and Energy Systems, vol. 37, no. 1, pp. $50-57,2012$.

[13] P. Roy, S. Ghoshal, and S. Thakur, "Optimal var control for improvements in voltage profiles and for real power loss minimization using biogeography based optimization," International Journal of Electrical Power and Energy Systems, vol. 43, no. 1, pp. 830 - 838, 2012.

[14] B. Venkatesh, G. Sadasivam, and M. Khan, "A new optimal reactive power scheduling method for loss minimization and voltage stability margin maximization using successive multi-objective fuzzy lp technique," IEEE Transactions on Power Systems, vol. 15, no. 2, pp. 844 -851, may 2000.

[15] W. Yan, S. Lu, and D. Yu, "A novel optimal reactive power dispatch method based on an improved hybrid evolutionary programming technique," IEEE Transactions on Power Systems, vol. 19, no. 2, pp. $913-918$, may 2004. 
[16] W. Yan, F. Liu, C. Chung, and K. Wong, "A hybrid genetic algorithm interior point method for optimal reactive power flow," IEEE Transactions on Power Systems, vol. 21, no. 3, pp. 1163 1169, aug. 2006.

[17] J. Yu, W. Yan, W. Li, C. Chung, and K. Wong, "An unfixed piecewiseoptimalreactive powerflow model and its algorithm for ac-de systems,"IEEE Transactions on Power Systems, vol. 23, no. 1 , pp. $170-176$, feb. 2008 .

[18] F. Capitanescu, "Assessing reactive power reserves with respect to operating constraints and voltage stability," IEEE Transactions on Power Systems, vol. 26, no. 4, pp. 2224-2234, nov. 2011.

[19] Z. Hu, X. Wang, and G. Taylor, "Stochastic optimal reactive power dispatch: Formulation and solution method," International Journal of Electrical Power and Energy Systems, vol. 32, no. 6, pp. $615-621,2010$.

[20] A. Kargarian, M. Raoofat, and M. Mohammadi, "Probabilistic reactive power procurement in hybrid electricity markets with uncertain loads," Electric Power Systems Research, vol. 82, no. 1, pp. $68-80,2012$.

[21] Zhou Xi-xiang, LiJia-sheng, Yang Sai-liang, The Digital PID Parameter Tuning Based on Chaos Particle Swarm Optimization, Power Electronics, 44(10): 62-64, 2010.

[22] Fei Kang, Junjie Li, Zhenyue Ma, Rosenbrock artificial bee colony algorithm for accurate global optimization of numerical functions, Information Sciences: S0020-0255(11)00198-8, DOI: 10.1016/j.ins.2011.04.024,2011.

[23] TatjanaDavidovic', Du`sanRamljak, Milica`Selmic', Du`sanTeodorovic', Bee colony optimization for the p-center problem, Computers \& Operations Research, 38(2011)1367-1376, 2011.

[24] IonáMaghali Santos de Oliveira, Roberto Schirru, Swarm intelligence of artificial bees applied to In-Core Fuel Management Optimizati, Annals of Nuclear Energy 38(2011)1039-1045,2011.

[25] D. Karaboga et al. "A comparative study of artificial bee colony algorithm". Applied Mathematics and Computation, 214(1):108-132, 2009.

[26] G. Zhu et al. "Gbest-guided artificial bee colony algorithm for numerical function optimization". Applied Mathematics and Computation, 217(7):3166-3173, 2010.

[27] J. H. Holland. "Outline for a logical theory of adaptive systems". Journal of the ACM, 3:297314, 1962.

[28] Talbi, El-Ghazali. "Metaheuristics: from design to implementation". Vol. 74. John Wiley \& Sons, 2009.

[29] Bansal, J. C. et al. "Information Sharing Strategy among Particles in Particle Swarm Optimization Using Laplacian Operator", Swarm Intelligence Symposium, 2009. IEEE, pages 30-36.

[30] Wright, "A. Genetic Algorithms for Real Parameter Optimization, Foundations of Genetic Algorithms", G. Rswlins (Ed.), Morgen Kaufmann publishers, CA, 1991, pp. 205-218.

[31] IEEE, "The IEEE 30-bus test system and the IEEE 118-test system", (1993), http://www.ee.washington.edu/trsearch/pstca/.

[32] Jiangtao Cao, Fuli Wang and Ping Li, "An Improved Biogeography-based Optimization Algorithm for Optimal Reactive Power Flow", International Journal of Control and Automation Vol.7, No.3 (2014), pp.161-176.

\footnotetext{
*Corresponding author.

E-mail address: gklenin@ gmail.com
} 